\title{
Temporomandibular Joint Ankylosis In Children
}

\author{
Mzubanzi Mabongo
}

\begin{abstract}
Temporomandibular joint (TMJ) ankylosis is defined as osseous or fibrous fusion of the condyle of the mandible and the mandibular fossa of temporal bone (Nitzan et. al. 1998).It is a debilitating disease that renders the afflicted person unable to use their oral cavity well. Clinical presentation depends on the age at which ankylosis occurs and whether ankylosis is unilateral or bilateral. Surgery and postoperative physiotherapy are regarded as the mainstay treatment for TMJ ankylosis.

The study reviews surgical outcomes of 13 children treated for temporomandibular joint (TMJ) ankylosis in the maxillofacial and oral surgery unit at Red Cross Children's Hospital. Nine patients were treated by gap arthroplasty, while four patients had previous reconstruction with costochondral graft.

One patient treated by gap arthroplasty reankylosed. The study showed a positive correlation between intraoperative $\mathrm{MIO}$ and the outcome, and between age and the outcome. Mouth opening was improved by $15 \mathrm{~mm}$, with postoperative change of $-4 \mathrm{~mm}$.
\end{abstract}

Key Words: TMJ ankylosis; maximal interincisal opening; gap arthroplasty; costochondral graft; interpositional arthroplasty and maximal interincisal opening (MIO)

\section{Literature Review}

Temporomandibular joint (TMJ) ankylosis is defined as osseous or fibrous fusion of the condyle of the mandible and the mandibular fossa of temporal bone ${ }^{1}$.TMJ ankylosis is a rare condition that renders the afflicted individual unable to masticate, articulate well and maintain good oral hygiene ${ }^{2,3,4,5}$. Some patients suffer from obstructive sleep apnoea due to small retruded mandible ${ }^{6}$. This condition deprives the afflicted person of good nutrition and opportunity to have proper dental treatment ${ }^{3,2}$. Inability to remove exfoliated teeth in children with TMJ ankylosis has been reported as well in patients with TMJ ankylosis ${ }^{7}$.

The incidence of TMJ ankylosis differs significantly in different parts of the world. Studies from Egypt $^{8,4,9}$ and India ${ }^{10}$ present with large samples, while studies from other parts of the world report fewer number of patients seen over a long period ${ }^{11,12,13,14}$. Kazanjian (1938) classified TMJ ankylosis according to the site involved into true (intracapsular) and false (extracapsular) ankylosis. Intracapsular ankylosis refers to fibrous or bony ankylosis that occurs between thecondylar head of the mandible and the mandibular fossa of the temporal bone. While extracapsular ankylosis refers to restriction of mandibular movement that occurs as result of pathology or physical obstruction that is outside the $\mathrm{TMJ}^{10}$.Intracapsular (True) ankylosis was further classified according to extent by Topazian and Sawhney ${ }^{15,16}$.

Aetiology of TMJ ankylosis may either be congenital or acquired. Acquired TMJ ankylosis may be caused by trauma, infection ${ }^{11,16,4,2}$, systemic inflammatory disorders ${ }^{17}$, irradiation, previous surgery ${ }^{2,18}$ and neoplasm $^{19}$. The posttraumatic TMJ ankylosis follows misdiagnosis, delayed treatment, inadequate surgery, prolonged immobilisation or insufficient physiotherapy ${ }^{20}$.

Most studies suggest that trauma is the most common cause of TMJ ankylosis ${ }^{8,20,12,6}$. In the preantibiotic era infection was the most common cause of ankylosis ${ }^{21}$. However Kaban et al 2009 maintain that infection is still the most common cause of ankylosis in the third world countries ${ }^{12}$. Some studies suggest that disc displacement is a prerequisite for posttraumatic ankylosis to occur. These authors maintain that this displacement allows a direct contact between the distal fragment of the mandible and the mandibular fossa or a clot occupies the region between the two surfaces ${ }^{15,1}$.

In children clinical presentation depends on the age at which ankylosis occurs, duration and on whether ankylosis is unilateral or bilateral ${ }^{22,12}$. When ankylosis occurs after growth has ceased, it presents mainly with limitation of mouth opening ${ }^{21,20}$. Currently, the surgical techniques used to treat TMJ ankylosis are gap arthroplasty, interpositional arthroplasty, joint reconstruction ${ }^{24}$ and distraction osteogenesis ${ }^{5,18}$. Some authors prefer to correct secondary deformities like facial asymmetry, occlusal canting and micrognathic mandible concomitantly with the release of the ankylosis $22,8,13$.

The goals for the release of TMJ ankylosis are to create a pseudoarthrosis that will improve function or movement of the mandible ${ }^{21,20,6}$, prevent relapse ${ }^{21}$, relieve airway obstruction if present ${ }^{8,2}$, achieve normal growth and correction of deformity in children ${ }^{6,12}$, restore appearance and occlusion in adults ${ }^{21}$ and facilitate maintenance of good oral hygiene. 
Reankylosis is the most common complication after surgery $y^{24}$. The reported causes of reankylosis are inadequate removal of ankylotic mass ${ }^{25,26}$ and lack of compliance to post-operative exercises ${ }^{20,27}$. Other complications that have been reported include profuse bleeding, transient facial nerve paralysis ${ }^{4,28}$ and sepsis ${ }^{4}$. While the following complication associated with use of costochondral graft are fracture of the costochondral graft (CCG) ${ }^{29}$, overgrowth of the graft ${ }^{30}$ and suboptimal growth ${ }^{31}$. Anterior open bite occurred in patients treated with gap arthroplasty $^{28}$.

Lello (1990) in his series of 13 cases that were treated by condylectomy, reconstruction with costochondral graft and use of interpositional material, only one case reankylosed ${ }^{32}$.

Guyuron and Lasa (1992) reported an unpredictable behaviour of costochondral graft, in 8 patients between 4 and 12 years, who had reconstruction with autogenous costochondral graft. In 4 patients there was excessive growth of the graft, 1 patient had sub-optimal growth and 3 patients had no growth ${ }^{33}$.

Manganello-Souza and Miriani (2003) reviewed 14 patients who were managed by interpositional arthroplasty. Recurrence occurred in one patient $(7.1 \%)^{23}$.

El-Mofty (1974) presented his results of 20 cases with unilateral TMJ ankylosis. Seventeen of these cases were 12 years or younger. All patients were treated by gap Arthroplasty. Recurrence occurred in 2 patients $(10 \%)^{4}$.Rajgopal et.al. (1983) achieved $100 \%$ success with gap arthroplasty ${ }^{28}$. Roychoudury et.al. (1999), also reported good results of 50 cases treated with gap arthroplasty. Mouth opening was improved in both children and adults, and reankylosis occurred in one patient $(2 \%)^{33}$.

These studies show that reankylosis is common complication irrespective of surgical technique used to treat the TMJ ankylosis.

The objective was to evaluate outcomes in children treated for TMJ ankylosis at Red Cross Children's hospitals; and to establish factors that influence the outcomes in Children.

\section{Study Design}

\section{Materials And Methods}

This is a retrospective study of the records of 13 patients treated for TMJ ankylosis in the Maxillofacial and Oral Surgery unit at Red Cross Children's Hospitals (RXH). All patients operated for TMJ ankylosis from January 1988 to March 2003 were included in this study.

\section{Surgical Data}

All patients were treated by the protocol described below:- Patients had Plain x-rays and computated tomography as standard imaging for confirming diagnosis of TMJ ankylosis.Preauricular incision was made. Excision of ankylotic mass. If adequate mouth opening was not achieved, ipsilateral coronoidectomy was done. Contralateral coronoidectomy via intra-oral approach (where necessary). When reconstruction was planned, costochondral graft was used. Modified submandibular incision was made. Stripping of the masseter from its insertion was done. Harvest of costochondral graft from rib 5,6 or 7. Costochondral graft was stabilised screws on the lateral aspect of the ramus of the mandible. Maxillary and mandibular alginate impressions were taken after the release of TMJs. After the operation mouth prop was used to keep the patients in maximum interincisal opening for $24 \mathrm{hrs}$. Exerciser was delivered $24 \mathrm{hrs}$ after surgery.

\section{Data Collection}

Clinical records were retrieved from RXH. Patients who were 12 years and younger during surgery were be grouped as peadiatric cases. Only patients with intracapsular ankylosis were included in the study. A ruler was used to measure maximal interincisal opening (MIO). The data that were analysed were categorised into pre-operative, intra-operative and post-operative phases. Preoperative data were analysed for age of the patient at the first visit, sex, aetiology, whether the ankylosis was unilateral or bilateral, type of ankylosis (fibrous or bony) and pre-operative maximal interincisal opening (pre-op MIO). Operative data included type of surgery, achieved intraoperative maximal interincisal opening (Op MIO) and intubation technique used. Postoperative data were reviewed for the outcome MIO (the last recorded maximal interincisal opening), complications and follow up period. In patients that were operated more than once, the data from the last operation was used.

In this study the outcomes were evaluated with reference to two parameters, namely pre-op MIO and Op MIO. Each of these variables was subtracted from the outcome MIO. A variable that uses pre-op MIO as a reference point is called an overall change in MIO (OC MIO) and one which uses Op MIO is called postoperative change in MIO (Pop MIO). OC MIO of $10 \mathrm{~mm}$ or more was regarded as good outcome. OC MIO less than $10 \mathrm{~mm}$ was regarded as reankylosis. 


\section{Ethical issues}

The study was approved by Research Committee of the University of Western Cape. Permission to use hospital records was gives by the hospital manager. Strict confidentiality was adhered to. Numbers were used to identify patients.

\section{Analysis of results}

Tables and graphs were used to demonstrate the outcomes. Statistica version 12 was used for statistical analysis of results.

\section{Results}

The age ranged between 4 years and 12 years. Seven of the patients were females and 6 were males. The aetiology of ankylosis was congenital, trauma and infection in 7,4 and 2 patients respectively. However in two patients that were thought to have a congenital cause of ankylosis, a condyle of the mandible was found lying on the medial aspect of the ankylotic mass. This was suggestive of the trauma as the cause of ankylosis.

\begin{tabular}{|l|l|l|l|l|l|l|l|}
\hline patient & Age & Sex & Aetiology & Side & Type & Airway & $\begin{array}{l}\text { Preop } \\
(\mathrm{mm})\end{array}$ \\
\hline 2. & 6 & Male & Tr (mva) & Unilateral & Fibrous & Competent & 8 \\
\hline 4 & 5 & Male & Congenital & Bilateral & Fibrous & Competent & 8 \\
\hline 6. & 12 & Female & Congenital & Unilateral & Bony & Competent & 20 \\
\hline 9. & 4 & Female & Congenital & Unilateral & Bony & $\begin{array}{l}\text { Tracheotomy } \\
\text { Dependant }\end{array}$ & 0 \\
\hline 10 & 5 & Female & Congenital & Bilateral & Bony & $\begin{array}{l}\text { Tracheostomy } \\
\text { Dependant }\end{array}$ & 14 \\
\hline 11. & 10 & Male & Congenital & Bilateral & Fibrous & $\begin{array}{l}\text { Tracheostomy } \\
\text { Dependant }\end{array}$ & 10 \\
\hline 18 & 9 & Male & Congenital & Bilateral & Bony & Competent & 1 \\
\hline 22 & 12 & Female & Tr. (mva) & Unilateral & Bony & Competent & 5 \\
\hline 27. & 6 & Male & Infection & Bilateral & Bony & Competent & 0 \\
\hline 28. & 5 & Female & Infection & Bilateral & Bony & Competent & 0 \\
\hline 29. & 12 & Male & Congenital & Bilateral & Bony & Competent & 0 \\
\hline 36 & 8 & Female & Trauma & Unilateral & Fibrous & Competent & 15 \\
\hline 45 & 4 & Female & Trauma & Bilateral & Bony & $\begin{array}{l}\text { Tracheostomy } \\
\text { Dependant }\end{array}$ & 8 \\
\hline
\end{tabular}

Table 1 shows preoperative data

TMJ ankylosis was unilateral in 5 patients and bilateral in 8 patients with total of 21 ankylosed joints. Four patients were tracheostomy dependant and 3 of these patients had bilateral TMJ ankylosis. Nine patients with intracapsular ankylosis were treated by gap arthroplasty, while four had grafting of the joint with costochondral graft. Both blind nasal intubation and fibre optic intubation were used in two and four patients respectively. While one patient was intubated by direct laryngoscopy.

Most patients had a negative postoperative change. This suggests that Op MIO was reduced, while few patients had a positive Pop MIO. Two patients reankylosed, both patients had unilateral bony ankylosis and were managed by gap arthroplasty. These two patients had a poor Op MOI of $15 \mathrm{~mm}$ and $20 \mathrm{~mm}$.

\begin{tabular}{|l|l|l|l|l|l|}
\hline Case & $\begin{array}{l}\text { Outcome MIO } \\
(\mathrm{mm})\end{array}$ & $\begin{array}{l}\text { OC } \\
(\mathrm{mm})\end{array}$ & $\begin{array}{l}\text { Pop } \\
(\mathrm{mm})\end{array}$ & MIO & Complication \\
\hline 2 & 33 & 25 & 8 & None & 37 \\
\hline 4 & 33 & 25 & 5 & None & 61 \\
\hline 6 & 27 & 7 & -13 & Reankylosis & 48 \\
\hline 9 & 0 & 0 & -20 & Reankylosis & 36 \\
\hline 10 & 26 & 12 & -4 & None & 66 \\
\hline 11 & 25 & 15 & -2 & None & 15 \\
\hline 18 & 26 & 25 & -2 & None & 60 \\
\hline 22 & 31 & 26 & 6 & None & 24 \\
\hline 27 & 10 & 10 & & None & 120 \\
\hline 28 & 10 & 10 & -5 & None & 132 \\
\hline 29 & 22 & 22 & -12 & None & 48 \\
\hline 36 & 34 & 19 & -1 & None & 106 \\
\hline 45 & 25 & 17 & -5 & None & 140 \\
\hline
\end{tabular}

Table 2 shows outcomes in the series 


\section{Discussion}

The study showed that aetiology was fairly distributed between trauma and congenital cases. Although the history suggested that congenital cases were more common, two cases with unilateral TMJ ankylosis whose aetiology was congenital according to history given, proved to be traumatic as the condyle was retrieved on the medial aspect of the ankylotic mass during surgery. This finding suggests that a unilateral congenital TMJ ankylosis should be confirmed during surgery by absence of a condyle. This finding supports the view of some authors who believe that the term "congenital ankylosis" tends to be applied to patients who had limitation of mouth opening at infancy which might have been caused by trauma during delivery ${ }^{15,28}$. However, El-Sheik (1999) applies the term congenital ankylosis to a condition where there is complete bony fusion between mandibular condyle and temporal bone, with absence of any trace of intra-articular disc and lateral pterygoid muscle ${ }^{8}$. This series showed that all congenital cases had bilateral TMJ ankylosis. This concurs with findings of Posnick and Goldstein $(1993)^{34}$.

Four patients in this series were tracheostomy dependent and three of these patients had bilateral TMJ ankylosis. TMJ ankylosis in these tracheostomy-dependent patients was either congenital or occurred in the first year of life. The airway compromise in these patients is due to relative normal growth of the tongue, within a confined space which is bounded by slow or non-growing mandible, resulting in the posterior displacement of the tongue ${ }^{35,8}$. This suggests that bilateral TMJ ankylosis that occurs very early in life is more likely to result in a compromised airway.

This series shows positive correlation between intraoperative MIO and the outcome MIO according to Spearman R (ref graph 1). This suggests that good Op MIO is one of the important factors that influence the outcomes in TMJ ankylosis. This concurs with Kaban et al 2009 who maintain that inadequate release of ankylotic mass is the main cause of reankylosis ${ }^{12}$.

\section{Correlations (Spreadsheet ankylosis.sta $\left.16 v^{*} 14 \mathrm{c}\right)$}

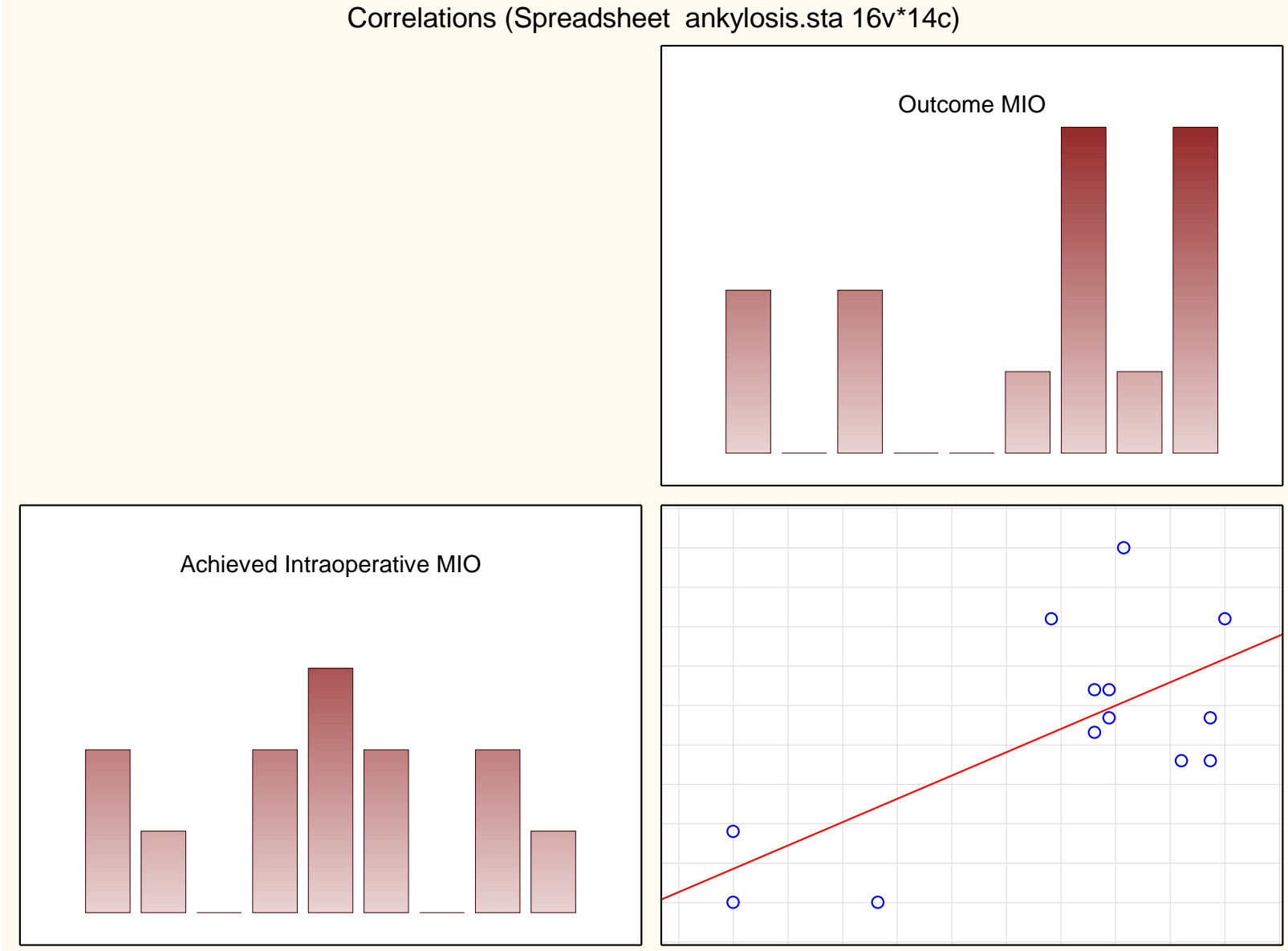

Graph 1: Scatter plot graph shows correlation between the intraoperative MIO and the Outcome

When the postoperative data was analysed, a mean postoperative progress shows a varying reduction of what was achieved intraoperatively with a mean of $-4,6 \mathrm{~mm}$. However the study shows a mean overall change of $15,2 \mathrm{~mm}$. 
The study also showed a positive correlation between the age and the outcome. This correlation was statistically significant with $\mathrm{p}<0,05$ (ref graph 2). The explanation for this positive correlation is that older patients understand the objective of the treatment, are more likely to cooperate with the exercises, resulting in good outcomes.In this series four patients had been previously reconstructed with costochondral graft and reankylosed. These patients were in their first decade during reconstruction. When reconstruction with costochondral graft was done for the second time, good results were obtained. It is assumed that these good results are achieved because patients in their teens understand the impact of the deformity and compliance to physiotherapy is better. This finding also supports authors who maintain that reconstruction with costochondral graft should be a secondary procedure after gap arthroplasty ${ }^{37,39}$.

\section{Correlations (Spreadsheet ankylosis.sta 16v*14c)}

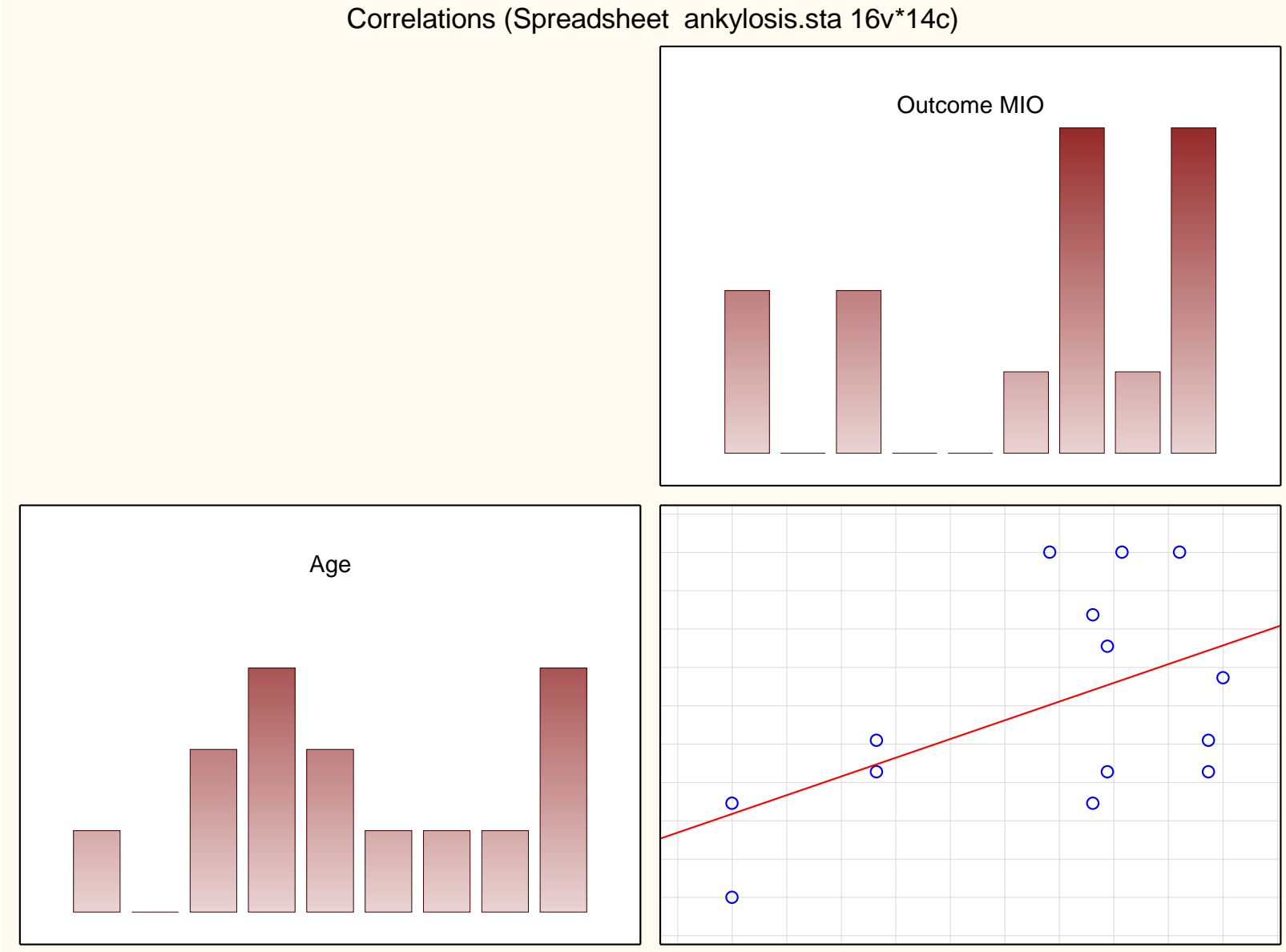

Graph 2: Scatter plot graph showing correlation between age of the patient and outcome

Literature suggests that costochondral graft (CCG) is a material of choice for reconstruction of TMJ in children $^{31}$. The advantages of CCG is that it offers three important functions ; cartilagenous portion act as an interpositional material ${ }^{24}$, the bony portion restores the vertical height of the ascending ramus ${ }^{24}$ and the junction between cartilaginous portion and the bony portion act as a growth centre ${ }^{36,35}$. The main objective of using costochondral graft in children is to transplant a growth centre ${ }^{36,34}$. The disadvantages of CCG are second operation site and donor site morbidity ${ }^{26}$ and unpredictable growth of the costochondral graft has been reported. Costochondral graft growth may be normal, suboptimal or may overgrow ${ }^{31}$.

These outcomes with costochondral graft concur with some studies that have reported poor results with costochondral graft ${ }^{38}$. Guyuron and Carlos (1992) reported unpredictability of costochondral graft as the main disadvantage because in their series of eight cases, overgrowth occurred in 4 cases, no growth in three patients and sub-optimal growth in one patient ${ }^{31}$. However, Lello (1990) reported good surgical outcomes with costochondral graft and interpositional material ${ }^{32}$.

Review of the literature shows that outcomes are mainly assessed by using the final IID, whereby outcomes are grouped into ranges of IID of $10-20 \mathrm{~mm} ; 20-30 \mathrm{~mm}$ and 30 and above ${ }^{15}$. Chessgros et al (1997) regard an IID of least $30 \mathrm{~mm}$ as a good result ${ }^{35}$, while Kaban et al (1990) believe an IID of $35 \mathrm{~mm}$ is a good outcome $^{26}$.However, Topazian (1966) regards an IID of $20 \mathrm{~mm}$ as adequate for function ${ }^{25}$. 


\begin{tabular}{|c|c|c|c|c|c|}
\hline Variable & No of cases & Mean & minimum & Maximum & STD Deviation \\
\hline Pre-op MIO & 13 & 6.4 & 0.0 & 20 & 6.6 \\
\hline Op MIO & 12 & 27 & 15 & 40 & 7.4 \\
\hline Outcome MIO & 13 & 21,6 & 0 & 34 & 11.8 \\
\hline Post op MIO & 13 & -4.6 & -20 & 8 & 8.5 \\
\hline OC MIO & 13 & 15.2 & 0 & 26 & 9.0 \\
\hline Follow up & 13 & 67 & 15 & 140 & 40 \\
\hline
\end{tabular}

Table 4 shows descriptive statistics of the outcomes of the study

This study introduces a new parameter in the study of outcomes in patients with TMJ ankylosis. This parameter, post-operative change in MIO (Pop MIO), actually shows the progress after surgery. It measures the actual impact of physiotherapy on the outcomes. Pop MIO can also be used in motivating and encouraging the patient to continue with the exercises and can also be used as a research tool. A positive Pop MIO should be a goal of every patient. The main disadvantage of this variable is that it cannot be worked out if intra-operative MIO was not recorded.

In the literature there is an agreement on the importance of aggressive physiotherapy after the release of TMJ ankylosis, but there is still controversy on when to start with the mobilisation of the jaws. Some authors believe in early mobilization ${ }^{17,33,28}$, while others immobilise the patient for a period of $1-10$ weeks $^{26,34,8}$. In this series an appliance was used for exercises.

The mean follow up time was 67 months with range of 15 to 140 months.(table 4)

\section{Conclusion}

This study suggests that there is positive correlation between preoperative MIO and intraoperative MIO and the outcome MIO.

\section{References}

[1]. Nitzan D.W.; Bar-Ziv B; Shteyer A 1998Surgical management of temporomandibular joint Ankylosis Type III by relating the displaced condyle and disc J. Oral Maxillofac. Surg. 56: 1133-1138

[2]. Elgazzar RF; Abdelhady AI, Saad KA et al 2010 Treatment modalities of TMJ ankylosis: experience in Delta Nile, Egypt Int. J Oral Maxillofac.Surg 39:333 - 342

[3]. Danda AK, Ramkumar S and Chinnaswami 2009Comparison of Gap Arthroplasty With and without a temporalis Muscle Flap for the Treatment of Ankylosis J Oral MaxillofacSurg 2009; 67:1425-1431

[4]. El-Mofty S 1974 Surgical treatment of ankylosis of the temporomandibular joint .J. Oral Surg. 32:202-206

[5]. Andrade NN, RaikwarKanchan R 2009 Management of Patients with Obstructive Sleep Apnoea Induced by Temporomandibular Joint Ankylosis: a novel 2-Stage Surgical Protocol and Report o 5 Cases. Asian J Oral Maxillofac Surg. 2009;21:27-32

[6]. Jain G, Kumar S, Rana Samar et al 2008 Temporomandibular joint ankylosis: a review of 44 cases Oral MaxillofacSurg 2008;12:61-66

[7]. El-Mofty S. 1972 Ankylosis of the temporomandibular joint Oral Surg. 33:650-660

[8]. El-Sheikh Mohammad M.1999 Temporomandibular joint ankylosis: the Egyptian experience. Ann. R. Coll. Surg. Engl. 81:12-18

[9]. SarmaU.C.and Dave P.K. 1991 Temporomandibular joint Ankylosis : An Indian experience Oral Surgery Oral Medicine Oral Pathology 72:660-664

[10]. Kazanjian, V.H.1938 Ankylosis of the temporomandibular joint Am. J. Orthodontia 24:1181-1206

[11]. Topazian R.G.1966 Comparison of gap and interposition athroplasty in the treatment of Temporomandibular joint ankylosisJ. Oral Surg. 24:405-409

[12]. Kaban LB, Bouchard C Troulis MJ 2009 A Protocol for Management of Temporomandibular Joint Ankylosis in Children J Oral MaxillofacSurg 67: 1966-1978

[13]. Feiyun P We L, Xin X, ZhuojinS et al 2010 Simultaneous correction of bilateral Temporomandibular joint ankylosis with mandibular micrognathia using internal distraction osteogenesis and 3-dimensional craniomaxillofacial models. J Oral MaxillofacSurg 68(3)571-7

[14]. Laskin D.M 1978 Role of the meniscus in the etiology of posttraumatic temporomandibular joint ankylosis Int. J. Oral Surg. 7:340-45

[15]. Topazian 1964 Etiology of ankylosis of temporomandibular joint: analysis of 44 cases J. Oral Surg. Anesth. \& Hosp. D. Serv., 22: $35-41$

[16]. Sawhney 1986 Bony Ankylosis of the Temporomandibular Joint: Follow-Up of 70 Patients Treated with Arthroplasty and Acrylic Spacer Interposition Plastic and Reconstructive Surgery 77: 29-40

[17]. Nwoku A.L. 1979 Rehabilitating children with temporomandibular joint ankylosis. Int. J. Oral Surgery 8:271-275

[18]. NitzanD.W.;AbuTair J; Lehman H 2012 Is entire removal of a post-traumatic Temporomandibular joint ankylosis necessary for an optimal outcome? J Oral Maxillofacial Surg. 70(12) 683-99

[19]. Su-Gwan K.2001Treatment of temporomandibular joint ankylosis with temporalis muscle and fascia flap Int. J.Maxillofac.Surg.189-193

[20]. Chidzonga M.M.1999 Temporomandibular joint ankylosis: review of thirty-two cases. Br. J. Oral Maxillofac. Surg. 37:123-126

[21]. Rowe N.L. 1982 Ankylosis of the temporomandibular joint J. of Royal Colleges of Surgeons of Edin. 27( P1); 67-79, (P2)167173, (P3) 209-218.

[22]. Munro I; Chen Y.R. and Park B. Y. 1986 Simultaneous Total Correction of Temporomandibular joint and Facial Asymmetry Plastic and Reconstructive Surgery 77(4):517-527

[23]. Manganello-Souza L.C;Mariani P.B. 2003Temporomandibular joint ankylosis: Report of 14 cases Int .J. Oral Maxillofac. Surg. 32:24-29

[24]. Kennett S 1973 Temporomandibular joint ankylosis: the rationale for grafting J Oral Surgery 31:744-748 
[25]. Topazian R.G.1966 Comparison of gap and interposition athroplasty in the treatment of Temporomandibular joint ankylosis J. Oral Surg. 24:405-409

[26]. Kaban L.B; D.H.Perrott and F.Fisher 1990 Protocol for management of temporomandibular ankylosis. J. Oral Maxillofac. Surg. 48:1145-1151

[27]. Andrade N N, KalraR,Shetye SP 2012 New protocol to prevent TMJ reankylosis and potentially life threatening complications in triad patients. Int. J Oral MaxillofacSurg 41:1495-1500

[28]. Rajgopal A.; BanerjiP . K.; BaturaV et al 1983 Temporomandibular Ankylosis: A report of 15 cases J. Max-fac. Surg. 11: 37-41

[29]. Merkx M.A.W. and Freihofer 1995 Fracture of costochondral graft in temporomandibular joint reconstructive surgery: an unexpected complication Int. J. Oral Maxillofac. Surg. 24: 142-144

[30]. Siavosh S and Ali M 2007 Overgrowth of a costochondral graft in a case of temporomandibular joint ankylosis J Craniofac Surg. 18(6):1488-91

[31]. Guyuron B and Lasa C.I.1992 Unpredictable Growth Pattern of Costochondral Graft Plastic and Reconstructive Surgery 90(5): $880-886$

[32]. Lello G.E.1990 Surgical correction of temporomandibular ankylosis. J.Cranio-Max-FacSurg 18:19-26

[33]. Roychoudhurry A; Parkash H; Trikha A. 1999 Functional restoration by gap arthroplasty in temporomandibular joint ankylosis: A report of 50 cases Oral Surgery Oral Medicine Oral Pathology 87(2); 166-169

[34]. Posnick J.C; J.A.Golstone 1993 Surgical management of temporomandilar joint in peadiatric population. Plastic and Reconstructive Surgery 5:791-798.

[35]. Chessegros C. L.; Guyot F; Cheynet, J.L et al 1997 Comparison of different materials for interposition arthropasty in treatment of temporomandibular joint ankylosis surgery: long-term follow-up in 25 cases.Br. J. oral and Maxillofac. Surg.35:157-160

[36]. Marx 1994 Clinical application of bone biology to mandibular and maxillary reconstruction. Clinics in Plastic Surgery 21:377 392

[37]. MacIntosh R.B. 2000 The use of autogeneous tissues for temporomandibular joint reconstruction. J Oral Maxillofac. Surg. 55:6369

[38]. Saeed N.R. and Kent . A retrospective study of the costochondral graft in TMJ reconstruction. Int. J. Maxillofac. Surg 2003;32: 606-609

[39]. Salins P.C. New perspectives in the management of craniomandibular ankylosis Int. J. Oral Maxillofac. Surg 2000; 29:337-340 